\title{
PaperIO: A 3D Interface towards the Internet of Embedded Paper-Craft
}

\author{
Kening $\mathrm{ZHU}^{\dagger \mathrm{a})}$, Nonmember, Rongbo $\mathrm{ZHU}^{\dagger \dagger b)}$, Member, Hideaki NII ${ }^{\dagger \dagger}{ }^{\dagger c)}$, Hooman SAMANI ${ }^{\dagger \dagger \dagger \dagger d)}$, \\ and Borhan (Brian) JALAEIAN ${ }^{+\dagger+\dagger+e), ~ N o n m e m b e r s ~}$
}

\begin{abstract}
SUMMARY As the development of Internet-of-Things is moving towards large scale industry, such as logistic and manifacturing, there is a need for end-users to get involved in the process of creating IoT easily. In this paper, we introduce PaperIO, a paper-based 3D I/O interface, in which a single piece of paper can be sensed and actuated at the same time in three dimensions using the technology of selective inductive power transmission. With this technology, paper material with multiple embedded receivers, can not only selectively receive inductive power to perform papercomputing behavior, but also work as input sensors to communicate with power transmitter wirelessly. This technology allows the creation of paperbased sensor and actuators, and forms an Interent of Embedded Paper-craft. This paper presents the detailed implementation of the system, results of the technical experiments, and a few sample applications of the presented paper-based 3D I/O interface, and finally discusses the future plan of this research.
\end{abstract}

key words: Internet of things, 3D user interface, tangible user interface, paper computing

\section{Introduction}

The Internet of Things (IoT) refers to the concept which entails the connection of everyday objects and devices to all kinds of networks such as company intranets, peer-to-peer networks and even the global internet, as defined by the International Telecommunication Union (ITU) in 2005 [1]. It is built upon embedded mobile technologies in a wide range of physical objects around us, enabling new forms of communication between human and things, and between things themselves. The rapid development on mobile technologies and embedded sensing technologies, such as smart phones, Radio-Frequency IDentification, embedded actuators, etc., indicated that the vision of IoT is no longer fictional. As the development of IoT is moving towards large scale of

Manuscript received December 23, 2013.

Manuscript revised July 10, 2014.

${ }^{\dagger}$ The author is with the School of Creative Media, City University of Hong Kong, Hong Kong.

${ }^{\dagger}$ The author is with the College of Computer Science, SouthCentral University for Nationalities, China.

${ }^{\dagger}+{ }^{\prime}$ The author is with the Research Laboratory, IIJ Innovation Institute, Inc., Tokyo, 102-0071 Japan.

${ }^{\dagger+\dagger}$ The author is with the Department of Electrical Engineering, College EE and CS, National Taipei University, Taiwan.

${ }^{+t^{+}+\dagger}$ The author is with the The Bradley Department of Electrical and Computer Engineering, Virginia Tech, USA.

a)E-mail: keninzhu@ cityu.edu.hk

b) E-mail: rongbozhu @gmail.com (Corresponding author)

c)E-mail: h-nii@iij.ad.jp

d)E-mail: hooman@mail.ntpu.edu.tw

e)E-mail: bran@vt.edu

DOI: 10.1587/transinf.2013THP0001 industry, including logistic, manufacturing, and health care, researchers argued that end-users need participate in the creation process of IoT, in order for the IoT to really take off. The users need to have the power and control over the creation and use of applications for IoT [2]. Dries De Roeck et al. [2] presented a manifesto for Do-It-Yourself (DIY) Internet-of-Things, as the guidelines for technologies that allows users to create their own IoT. The manifesto indicated that a Do-it-Yourself internet-of-things creation system should be easy in technologies and materials, inspiring people to create ideas and solutions, allowing the use of multimodel input, using body and objects.

Among low-cost materials, paper stands out because of its unique advantages: it is easy to use, flexible, lightweight, and readily available. As a traditional medium for art and communication, it has long been popular for creating 2D and 3D models as well as paper craft. In addition, researchers in organic user interface (OUI) [3] argued that an OUI is a user interface with non-planar displays that actively or passively change shape via analog physical inputs. Therefore, we can see that a single piece of paper or paper-craft objects can be developed as a stand-alone organic user interface, and connected to the networks to form the internet of embedded paper-craft, which is affordable for taking any tangible input, such as folding and cutting, and also to transfer these inputs into different shapes as outputs through the networks. In addition, researchers who are interested in paper material to bring up the concept of Paper Computing [4], which explored paper as input or output in human computer interaction. However, few of them allow paper to both control and be controlled physically by digital contents at the same time, which separated the usage of paper as input and output in different contexts.

Motivated by the manifesto of DIY internet-of-things and the concept of Internet of Embedded Paper-craft, this paper presents PaperIO, a new type of paper-based I/O interface based on the technology of selective inductive power transmission [5]. It is able to selectively provide inductive power to different receivers in the context of multiple receivers embedded in paper material, and further actuate the shape-changing of paper as an organic output method. Based on this technology, we developed a technique to enable power receivers to send feedback back to power transmitter while receiving power wirelessly at the same time, thus allow the system to sense the 3D information (Here we define $3 \mathrm{D}$ information as the presence, the positions and the 


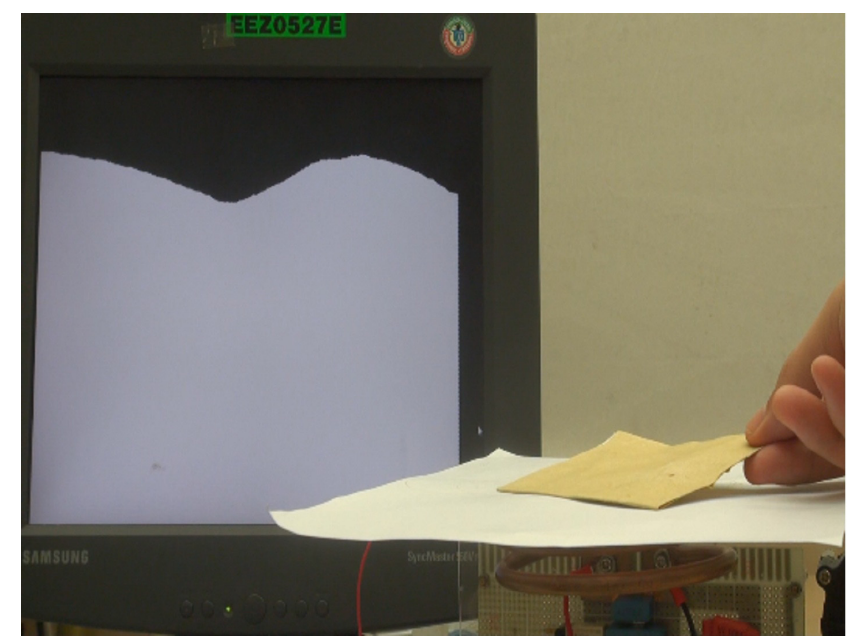

Fig. 1 PaperIO Interface: paper material integrated with receiving coil causes the change of the sensor output.

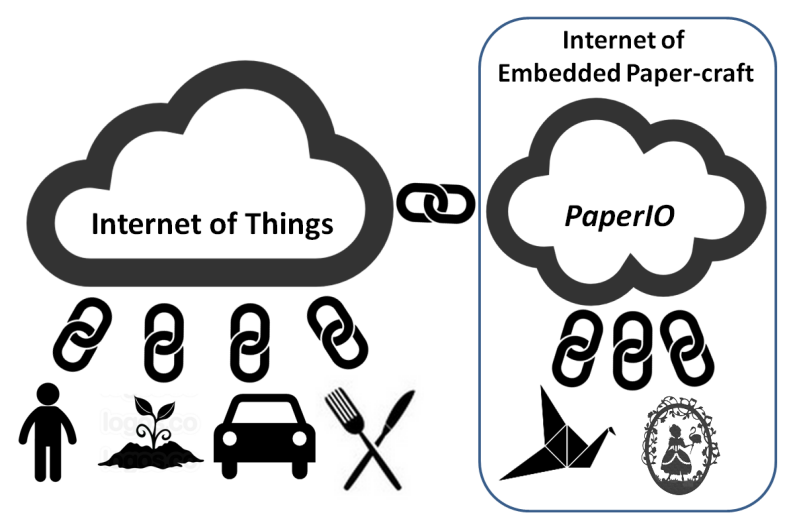

Fig. 2 PaperIO links paper-craft to Internet of Things, and creates Internet of Embedded Paper-craft.

orientations) of power receivers, as shown in Fig. 1, integrating both output and input interfaces in paper material with embedded power receivers.

As shown in Fig. 2, PaperIO creates the connection of paper-craft and internet, and further forms an Internet of Embedded Paper-craft. Compared to existing research, PaperIO doesn't required complex electrical sensor and actuator circuit embedded in the paper where power receivers are the sensors themselves, and it is real-time and camera-free to avoid the problems such as lighting and hand occlusion. In the rest of this paper, an overview and comparison of the related work in paper input and output will be presented and discussed in the next section. As follow, the detailed implementation of PaperIO will be presented with experimental results of technical performance. Then a few examples of interaction will be described as using PaperIO as a 3D input interface for pure virtual reality, and using PaperIO as both input and output interfaces at the same time for paper-based interaction. Finally, the paper concludes with the discussion and the future work of PaperIO.

\section{Related Work}

PaperIO falls on the general research of Paper Computing, which using the 3D information of paper as input and output interfaces. This section will review and compare PaperIO with these related works.

\subsection{Paper-Craft as Input}

The presented system is highly related to using paper material as an input medium based on hardware implementation. In order to overcome the shortage of computer-vision-based paper input, researchers started to explore the hardwarebased solution for 3D paper input. RFID [6] is one of the earlier hardware-based paper input technologies. However, it can only sense the presence of the paper-like tag, instead of more advanced information such as position and orientation. Yingdan Huang et al. [7] developed Easigami, a tangible user interface which embeds potentiometers on the edge of paper, so that users can construct different shapes of paper models by combining paper which is then reflected in a 3D virtual representation. In Pulp-based Computing [8], Marcelo Coelho et al. developed a paper-based bending sensor by infusing carbon resistive ink in between two layers of paper. Therefore, the resistance of the paper bending sensor would change while the paper is being bended. Byron Lahey et al. [9] developed PaperPhone, which is the combination of a flexible E-Ink display and a bending sensor connected to the external processing circuit. Paper-Phone allows users to navigate the digital content through a set of pre-defined bending gestures.

While the existing works in hardware-based paper input required complex processing circuit either embedded in or connected to the sensors and the paper material, PaperIO doesn't contain any complex circuit in the paper, besides the flat power receiving coils which are providing the 3D input. This fact simplifies the design of PaperIO sensors, and makes it possible for end-users to design and implement their own PaperIO sensors.

\subsection{Paper-Craft as Output}

PaperIO is also motivated by how to actuate paper output automatically. Greg Saul et al. [10] developed a set of interactive paper devices with embedded electronics, which can actuate light, sound and movement in paper. In these paper devices, conductive paths are inkjet-printed on the paper to form the complex schematics of circuits, and electrical components, such as microprocessor, speaker, and shape memory alloy, are soldered to the conductive paths. In Pulpbased Computing, the circuits are screen-printed on the paper material with components embedded during pulp making process. Using Pulp-based Computing and LilyPad [11], Jie Qi et al. developed Electronic Popables [12], the interactive pop-up books that integrates electronics and pop-up 
mechanisms as a complete user interface. Animated Paper [13] was presented as a versatile platform created from paper and shape memory alloy (SMA), which is easy to control using a range of different energy sources from sunlight to lasers. In Animated Paper, the paper craft attached with SMA can be controlled to move with a high-power laser pointer. More recently, AutoGami [14] was developed as one step forward for allowing end-users to create their own automated movable paper-craft.

In summary, there are three main contributions we claimed for PaperIO technology over existing paper interfaces. Firstly, compared to computer-vision-based paper input, PaperIO doesn't rely on any light condition, and it allows users to manipulate the paper with embedded receivers with hands directly and freely. These features overcome the disadvantage of lighting and hand-blocking. Secondly, while comparing with most of the existing hardware-based solutions for paper input and output, PaperIO utilizes the technology of selective inductive power transferring, and only simple coils are embedded in paper material. We believe these could enhance the tangibility and the customizability of the paper interface. Finally, in the technical aspect, PaperIO could provide a wide range of variations in the input of 3D information, including gradual changes in position and orientation, as shown in the experiments.

\section{System Description}

\subsection{Overview of Theoretical Principle}

The basic principle of PaperIO is based on the theory of inductive power transmission. As shown in Fig. 3, the power receiving coil is connected to a capacitor $\mathrm{C} 1$, which tunes the resonant frequency of the receiving coil to a specific value, and a diode D1, which will be turned on only when the receiving coil is closed enough to the power transmitter for receiving enough power. When D1 is turned on, the neg-

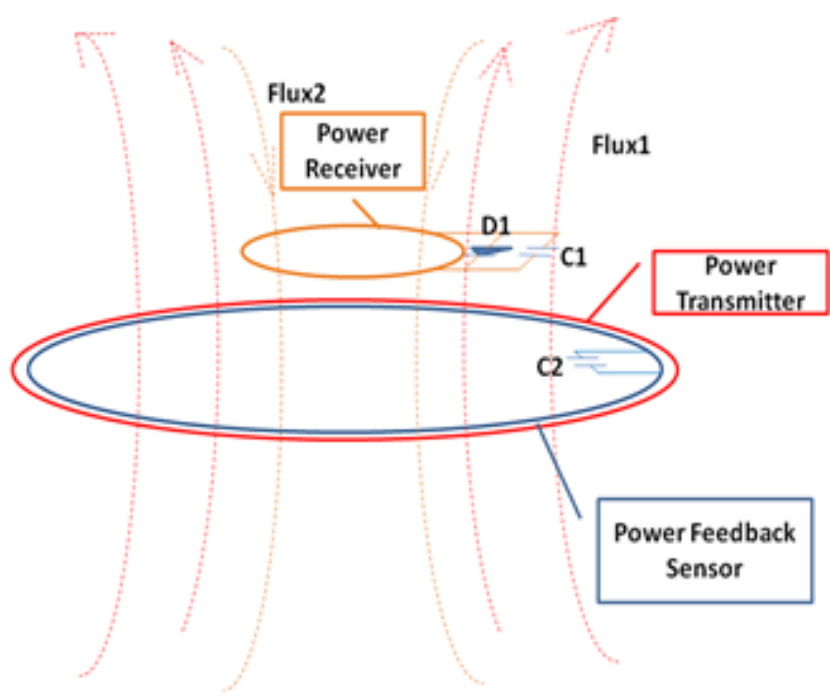

Fig. 3 Basic theoretical principle of PaperIO. ative part of the $\mathrm{AC}$ current in the receiving coil would be cut-off, and the receiving coil generates a new magnetic flux with almost twice of its resonant frequency. Therefore, the power feedback sensor with the capacitor $\mathrm{C} 2$ could sense the change of the magnetic flux, as its resonant frequency is tuned by $\mathrm{C} 2$ to the twice value of the resonant frequency of the power receiving coil.

The magnetic flux generated by the power receiver (Flux2 in Fig. 3) would change when the power receiver's position and orientation are changed. Therefore, this sensor should be able distinguish the difference when the receiver is moving, and further enable it as a $3 \mathrm{D}$ input device. In addition, by controlling the value of $\mathrm{C} 2$, the power feed-back sensor can have multiple resonant frequencies to differentiate the presence of various power receivers. Finally, receivers with simple actuators, such as shape memory alloy, embedded in paper material, make paper as both input and output devices.

\subsection{Simulation}

Based on the theoretical hypothesis described above, we first simulated PaperIO in SPICE software environment [15]. As shown in Fig. 4, there are three resonant circuits in the schematic: transmitter (L1), receiver (L2) and feed-back sensor (L3), while L1 and L2 are linked by coupling coefficient K1, L2 and L3 are linked by coupling coefficient $\mathrm{K} 2$, and L1 and L3 are linked by coupling coefficient K3. On the left side of the simulated schematic, the transmitting coil is excited by a sinusoid power source with the amplitude of $12 \mathrm{~V}$. For the purpose of simulation, we picked 510 $\mathrm{kHz}$ for the value of transmitting frequency. The transmitter is modeled as a two-turn coil (L1) with the diameter of $10 \mathrm{~cm}$ and the resistance (R1), and the feedback sensor is modeled as a two-turn coil (L3) with the diameter of $5 \mathrm{~cm}$ and the resistance (R2), and placed $2 \mathrm{~mm}$ above L1. The right side of the schematic shows the model of power receiver, which is a 2-turns circular coil with the diameter of 5 $\mathrm{cm}$, and it is connected to diode D1 and capacitor $\mathrm{C} 1$ for the resonant frequency of $510 \mathrm{kHz}$. In this model, we assume the closest distance between L1 and L2 is $1 \mathrm{~cm}$. According to the equations in [5], we calculated the values of L1, L3 and $\mathrm{K} 3$ as shown in Fig. 3. Additionally, Fast Fourier Trans-

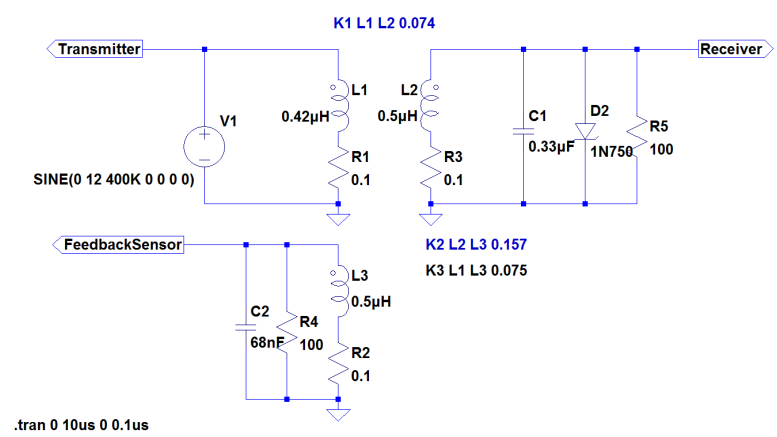

Fig. 4 Simulation schematic for PaperIO. 


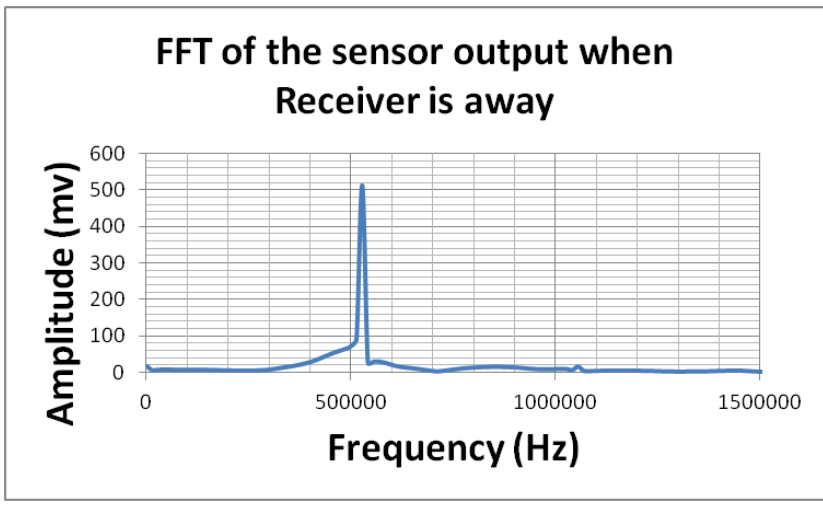

(a)

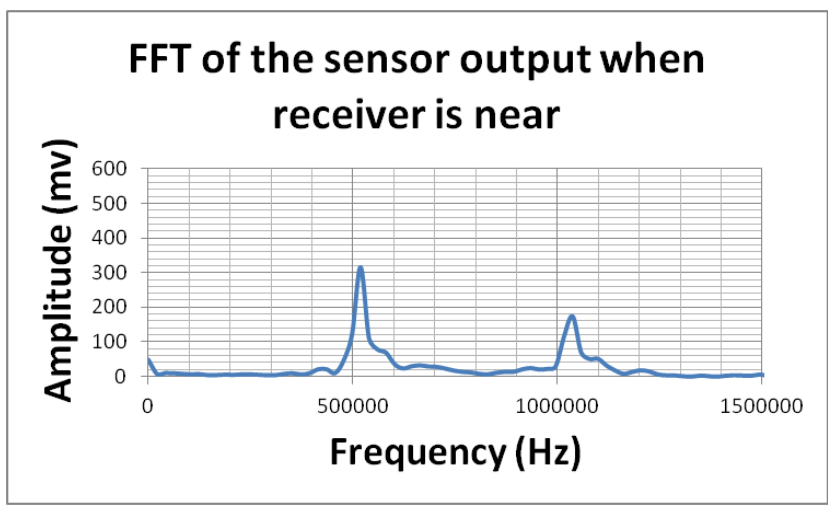

(b)

Fig. 5 (a) The FFT result of sensor signal when the receiver is away; (b) The FFT result of sensor signal when the receiver is near.

form (FFT) was employed to process and analyze the raw signal from the feedback sensor in the frequency domain in the simulation.

When the receiver is not present near the transmitter, there is only one active coupling $\mathrm{K} 3$ between the feedback sensor and the transmitter. The simulation results of this situation are shown in Fig. 5 (a), where the signal detected by the sensor only shows one peak value in the frequency domain at the frequency of $510 \mathrm{kHz}$. When the receiver is moving closer to the transmitter and the sensor, the coupling K1 and K3 are activated. The simulated results (Fig. 5(b)) show that different values of amplitude occur in the frequency domain at the value of $1.02 \mathrm{MHz}$, which is twice of the value of the resonant frequency of the receiver.

When the values of $\mathrm{K} 1$ and $\mathrm{K} 2$ are changed, due to the change of the receiving coil's position or orientation, the amplitude at the frequency of $1.02 \mathrm{MHz}$ would also change. Therefore, we can develop a circuit prototype to detect this value-changing in the frequency domain, and further map this change to the $3 \mathrm{D}$ information of the power receiving coil.

\subsection{Hardware Implementation}

Based on the theoretical hypothesis and the software simulation, we designed implemented the hardware prototype for

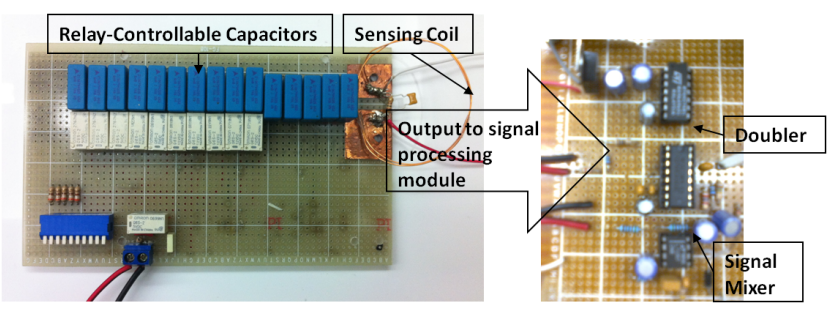

Fig. 6 Prototype of PaperIO sensing coil with controllable capacitors.

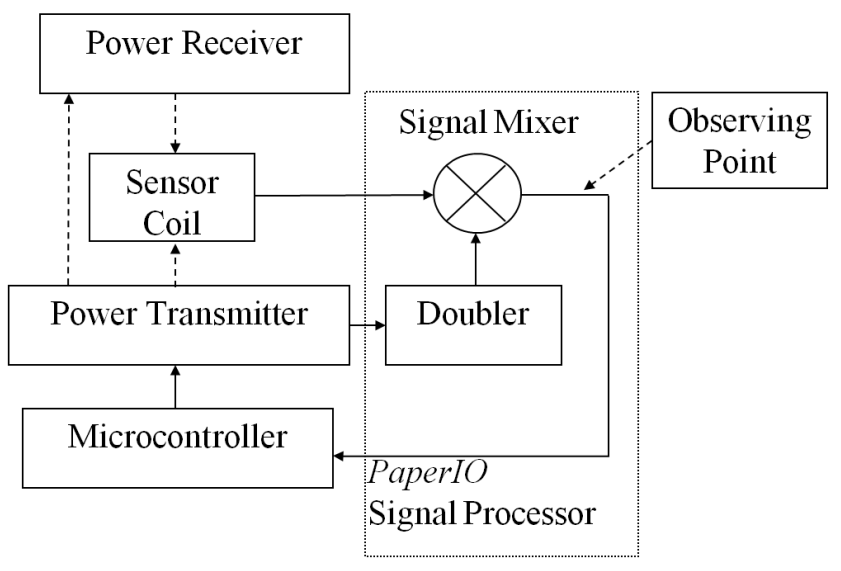

Fig. 7 System diagram of PaperIO.

detecting power feedback signal for PaperIO. For the first prototype of the feedback sensing coil, we designed its dimension similar to the power receiving coil with the diameter of $5 \mathrm{~cm}$, as shown in Fig. 6, and it is installed $2 \mathrm{~mm}$ above the transmitting coil. In order to detect different receiving coils in different frequency, the sensing coil is connected to an array of capacitors which would be controlled to switch on or off by the relays, to achieve various resonant frequencies.

In addition, a noise-reducing module is required in order to increase the robustness and the accuracy of the sensing coil. In the system diagram in Fig. 7, the feedback sensor coil receives two different signals from the transmitting coil and the receiving coil, and only the signal with two-times frequency from the receiving coil is useful for the $3 \mathrm{D}$ input detection. Therefore, we designed the PaperIO Signal Processor module, as the core of the hardware system, to reduce the noise signal from the transmitting coil.

The PaperIO Signal Processor module contains two main units: the frequency doubler and the signal mixer. As the raw signal from the sensing coil is a mixed signal with the frequency whose value is twice of the transmitting frequency, a double-frequency signal is required, in order to reduce the noise using digital operational mixer circuit. The frequency doubler is designed based on the theory of PhaseLocked Loop (PLL), and the doubled signal is passed to one input of the signal mixer which is an operational subtractor, while the other input signal is the raw signal detected by the feedback sensing coil. The operatoional subtractor is built with an Resistor-Capacitor-based low-pass filter, to filter the 
high-frequency part of the signal from the doubler. Finally, the output result from the signal mixer is the signal detected only from the power receiving coil. Furthermore, the denoised signal is passed to the anlog-to-digital module of the microcontroller to control the power transmitter.

\section{Performance Experiments}

\subsection{Experiment Design}

The output from the PaperIO sensor is passed to the analog input port of Arduino processor, and the method of Simple Moving Average (the sample window length is 50) is used to further reduce the noise of the output signal, and the moving-averaged signal is normalized into the scale from 0.0 to 1.0 , and finally output to the serial port of the Arduino processor. During the experiments, we fixed the transmitting frequency at $510 \mathrm{kHz}$ as the proof of concept for the receiver, as shown in Fig. 8.

\subsection{Initial Test: Presence of Receiver}

For the first test of the PaperIO sensing system, we experiment the sensitivity of whether the receiving coil is directly placed closely on the power surface without any user manipulation. In the experimental setup, the closest distance between the transmitter and the receiver is $1 \mathrm{~cm}$, in order to avoid over-large power and protect the components in the receiving coil.

Figure 9 summarizes the results of this initial experiment. When the receiver is placed $1 \mathrm{~cm}$ above the power transmitter, it can be observed that the presence of receiver can be clearly distinguished by the dropping of the sensor output, due to the feedback double-frequency signal from the power receiver. The value of the sensor output rises when we take away the receiver. Additionally, the transient slope during placing the receiver inspired us to experiment the sensitivity of different position of the receiver.

\subsection{D Input: Horizontal Translation}

In the experiments of sensing the horizontal translation of the receiver, the target was to find out whether the sensor output would change when the receiver is moved on the horizontal level. The receiver was placed at the starting 1 positions not aligned with the sensor, as shown in Fig. 10.

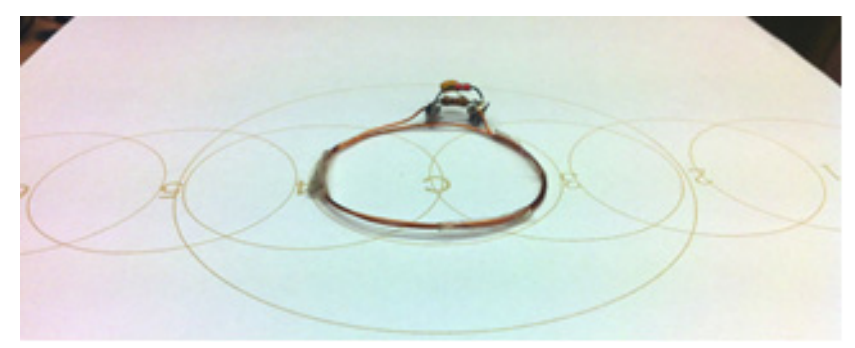

Fig. 8 Power receiving coil directly placed close to the power surface.
During this experiment, the receiver was placed closed to the transmitter, and moved from the position 1 to position 6 through the center position for the distance of $15 \mathrm{~cm}$, and the sensor output was recorded accordingly.

The results in Fig. 11 show that the sensor gives different output when the receiver is at different horizontal positions. When the receiver is moving from center to the other position, the transient pattern of the sensor output can be recorded as the input for interactive systems. To be noted specially, when the receiver reached position 2 and position 5 , the sensor output increased to higher than the output when the receiver was not presence, such as position 1 and position 6 .

\subsection{D Input: Vertical Translation}

For the experiment of moving the receiver vertically, we aimed to determine to which height the receiver can be sensed by the sensor and different sensor output for different height of the receiver. The set-up of the experiment is shown in Fig. 12. The acrylic box contains slots for inserting a flat and thin plastic sheet to hold the receiver at a certain height, and the vertical distance between two nearby slots is $5 \mathrm{~mm}$. This structure allowed us to control the verti-

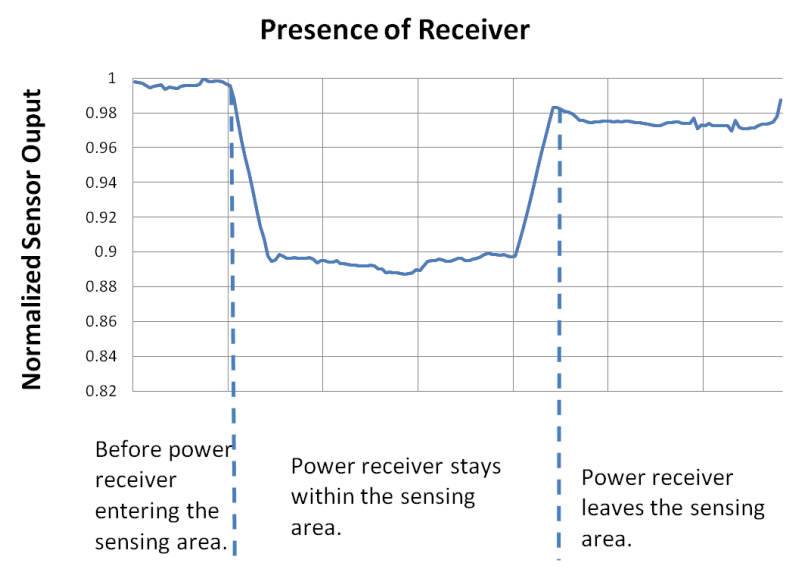

Fig. 9 Experiment results of the presence of receiver.

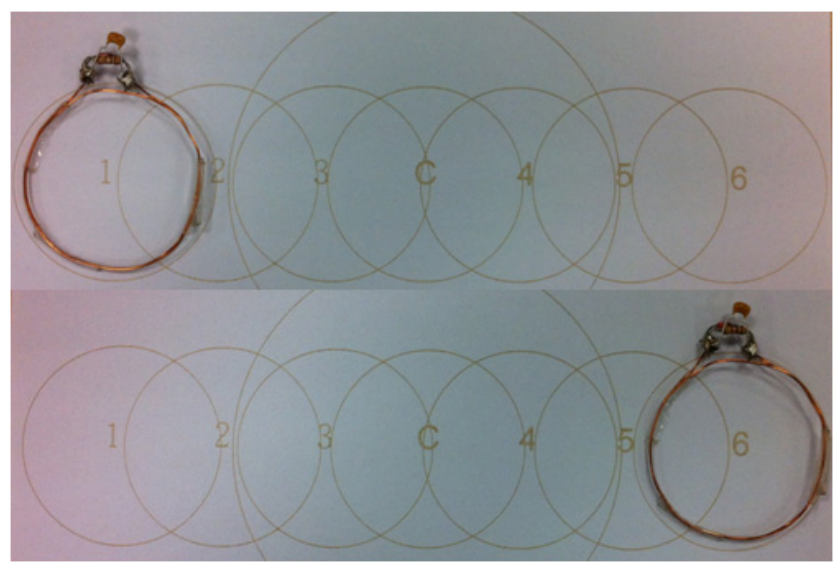

Fig. 10 Experiment to detect the horizontal movement of receiver. 


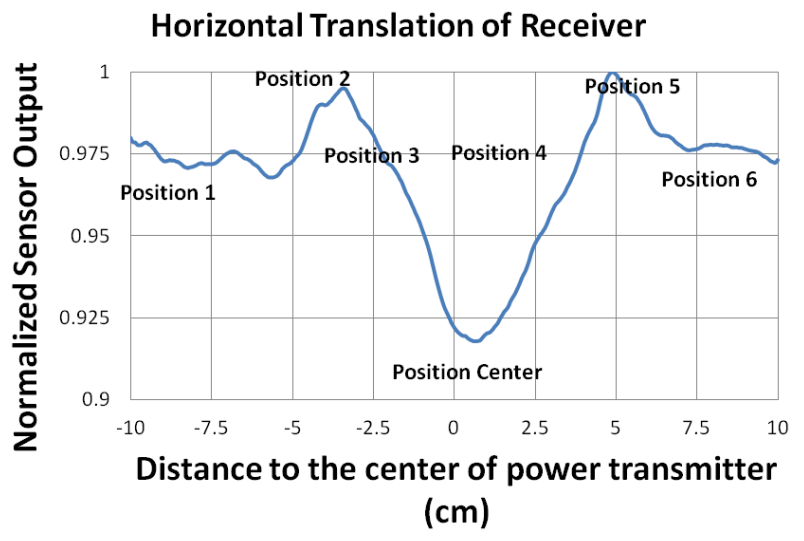

Fig. 11 PaperIO sensor output of the horizontal movement of receiver.

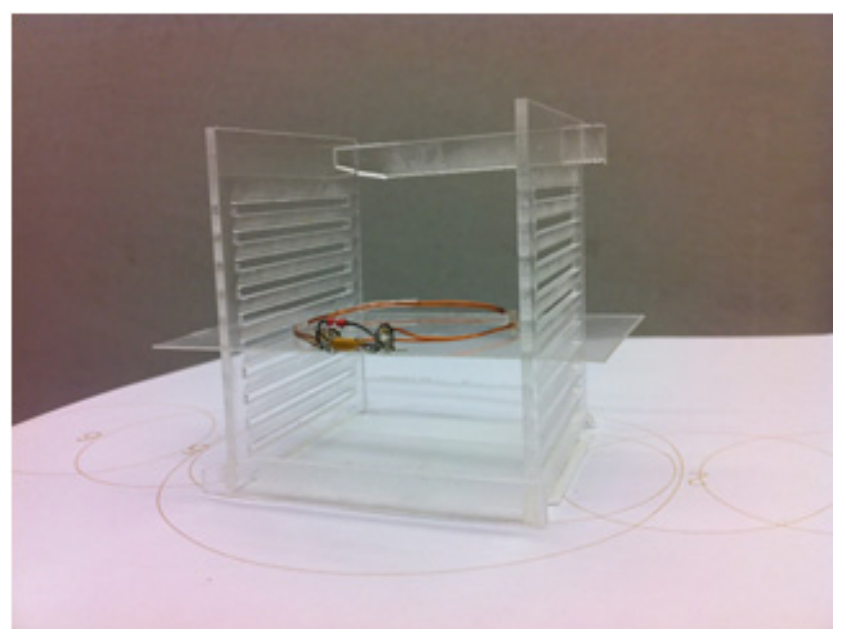

Fig. 12 Experiment setup for sensing vertical translation of receiver.

cal distance between the receiver and the sensor. When the distance was changed, the receiver was removed from the scene and placed back after changing the distance. In this experiment, the vertical distance was changed from $0 \mathrm{~mm}$ to $20 \mathrm{~mm}$.

Figure 13 illustrates the results of sensing the vertical translation of the receiver. The normalized sensor outputs were clearly distinguished as the receiver was placed at different height above the sensor. Therefore, the concept of using PaperIO as a height sensor is proofed.

\subsection{D Input: Orientation}

For the setup of sensing the orientation angle of the receiving, a set of angle models with the specific values of $5^{\circ}, 10^{\circ}$, $20^{\circ}$, and $30^{\circ}$ were made using acrylic material as shown in Fig. 14, and other values of angle can be realized by stacking these models together. During this experiment, the angle model was placed on the surface right above the sensor, and the receiver was fixed on the model as shown in Fig. 14. The model would be changed from $0^{\circ}$ to $60^{\circ}$, in order to measure sensor output in different values of orientation angles of the receiving coil.

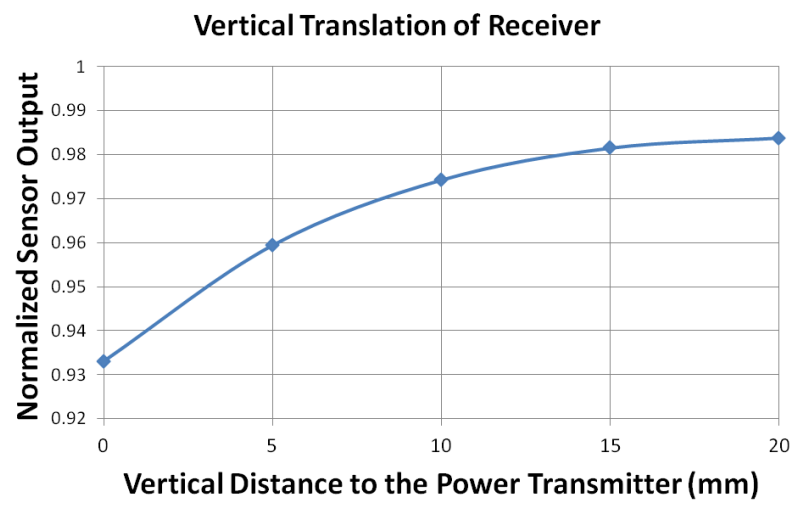

Fig. 13 PaperIO sensor output of the vertical movement of receiver.

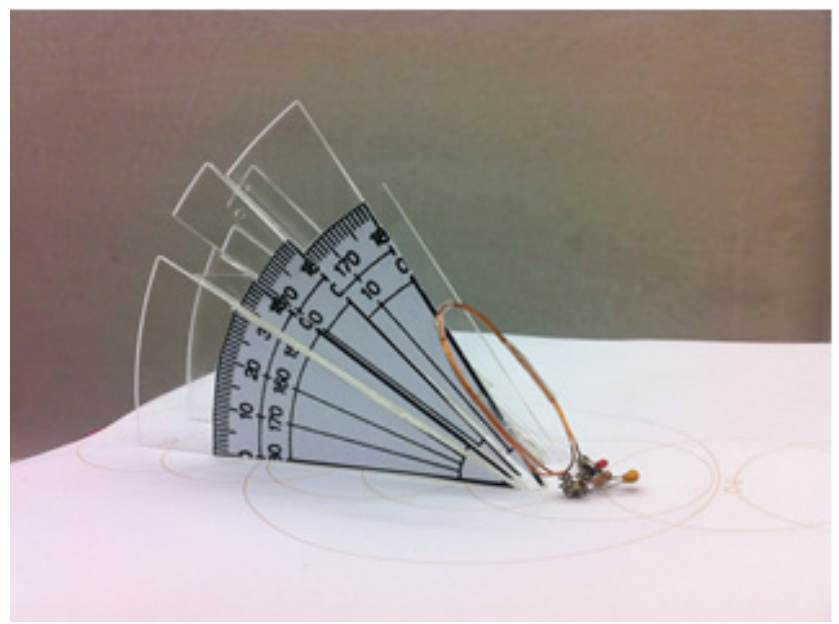

Fig. 14 Experiment setup for sensing orienta-tion of receiver.

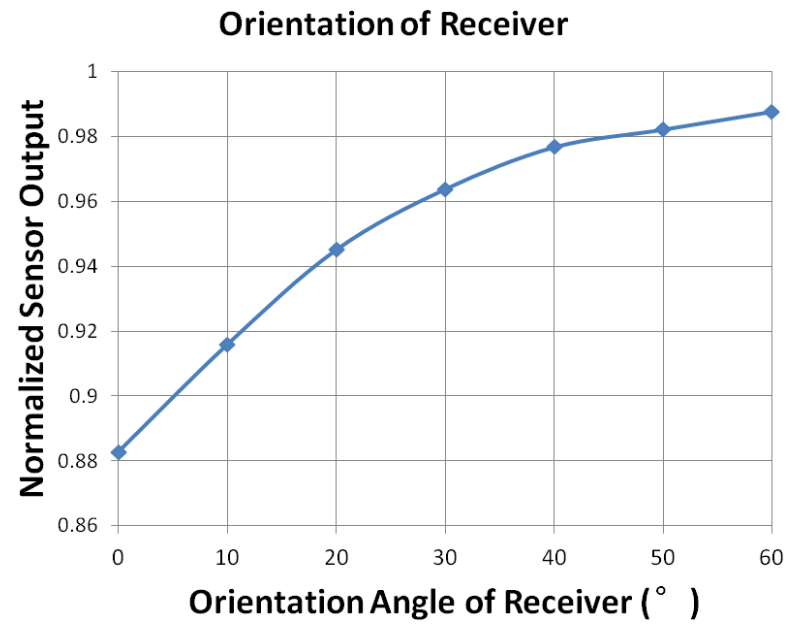

Fig. 15 PaperIO sensor output of the tilting of receiver.

The experimental result, as illustrated in Fig. 15, shows that the output value of the sensor decreases when the receiver is present near the transmitter, and the output value increases along with the increase of the angle between the receiver and the transmitter. This provides a proof of con- 
cept that PaperIO can also be used as an orientation input.

In summary of the series of experiments for 3D information input using PaperIO technology, PaperIO is able to provide the distinguishable analog output continuously according to the receiving coils' positions and orientations while it doesn't require explicit wire connection to the power receivers. Due to the flatness of the power receiving coils, PaperIO technology can be easily integrated with paper material to facilitate paper-based human computer interaction.

\section{Examples of Application}

For the application of PaperIO sensor, we focused in paperbased ubiquitous computing, mainly daily activities, gaming entertainment, and social networking. As the proof of concept, we designed three examples of the interaction, Paper Controller for Virtual Reality, Paper-based Digital Bookmark, and Origami Interaction.

\subsection{Paper Controller for Virtual Reality}

One direct application for PaperIO is embedding the receiver with pulp material as an integrated controller of paper. Therefore, the manipulation of the integrated paper, such as tilting, lifting, flipping, could be directly mapped to the motion of the virtual objects. An simple example is shown in Fig. 16 that the manipulation of paper is directly reflected by the virtual flat plane. This interaction allows users to use paper material directly as the controller for navigating and manipulating virtual reality, such as gaming.

\subsection{Paper-Based Digital Bookmark}

As the PaperIO could indicate different height of the receiver, the paper sheet integrated with receiver could be inserted into a physical book as a piece of bookmark, to indicate the current reading progress, and connect the physical book with digital book library. The receiver with the

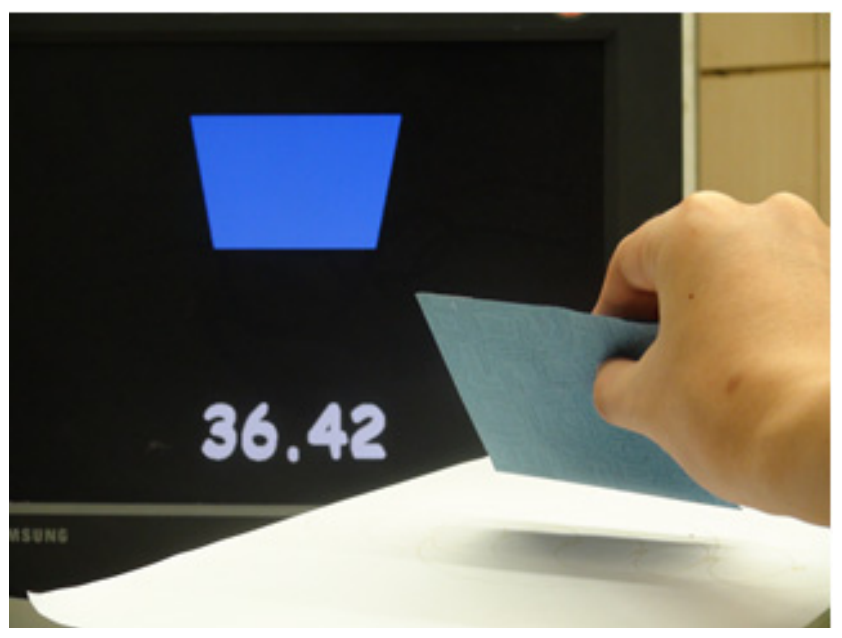

Fig. 16 Manipulate virtual object with PaperIO. resonant frequency of $510 \mathrm{kHz}$ is fixed to the first page to indicate the total height of the book, and the other receiver, with the resonant frequency of $322 \mathrm{k}$, can be inserted in any page in the book (Fig. 17). When the user puts the book near the power transmitter, PaperIO sensor reads the distances of both receivers, and calculates the ratio of these two values, to show the progress of book reading, as shown in Fig. 18.

\subsection{Physical Origami Interaction}

The ability of detecting the orientation of receiver makes $\mathrm{Pa}-$ perIO a origami-folding interface. As shown in Fig. 19, the PaperIO sensor can detect the folding of one corner which contains a receiving coil being tilted due to the folding, and control the power transmitter to provide power to the receiver in the other part of the paper, to activate the connected shape memory alloy and trigger the automatic paperfolding. This origami interaction provides users a physical and tangible feedback of origami making, and gives the possibility of physical creative interface for origami co-

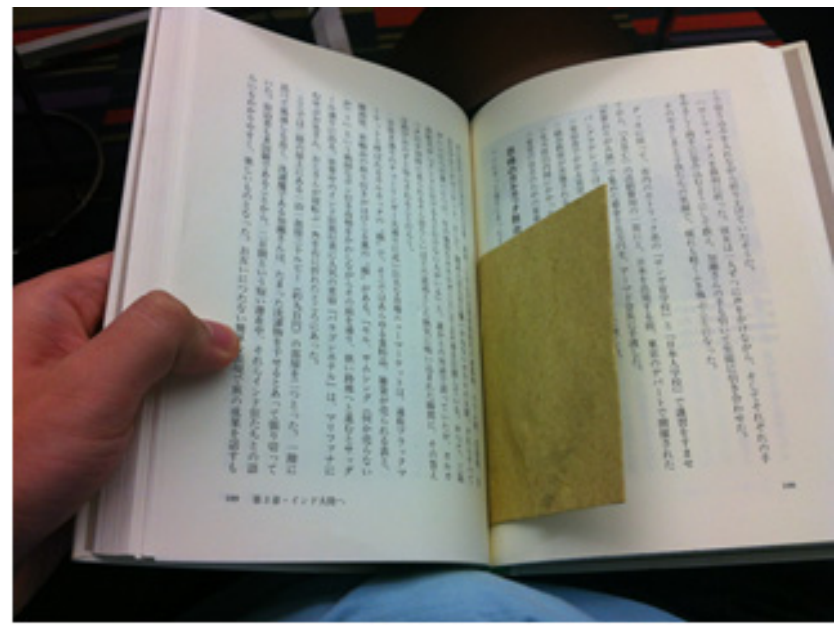

Fig. 17 Using PaperIO as a bookmark.

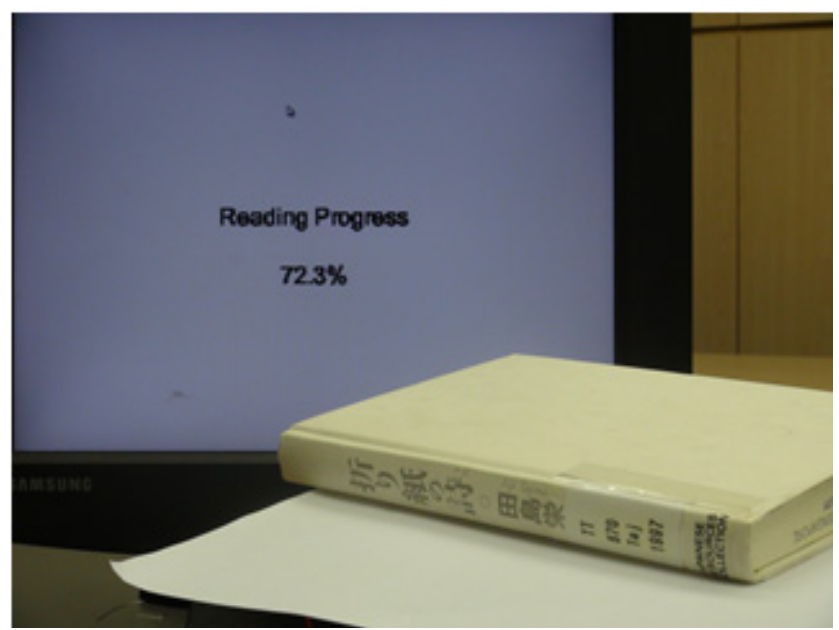

Fig. 18 PaperIO senses the reading progress of the book. 


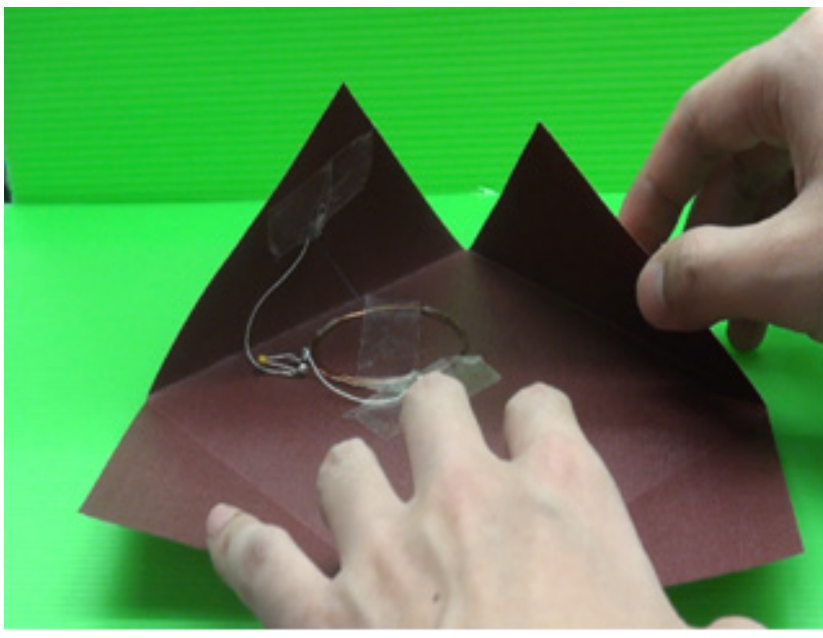

Fig. 19 Paper-folding sensor to trigger automatic folding.

designing between human and computer.

\section{Future Work \& Conclusion}

Since PaperIO is in the early stage of prototyping for the proof of concept, there are still a few limitations and space for improvement in the current system. Firstly, although the experiments showed that PaperIO could provide a quite accurate results for the $3 \mathrm{D}$ information of the power receiving coils, the results are still affected by the environmental noise, either from the peripheral electromagnetic signals or from the unstable hand movements by the users. Therefore, a better noise-reducing module is required for more accurate results from the PaperIO system. Secondly, it is difficult for the current version of PaperIO to work as translation input and orientation input at the same time. Therefore, advanced interaction skills would be employed to support PaperIO interface. Finally, we will measure the change of power transmission along with the change of the $3 \mathrm{D}$ information of the power reciever, to further study the $\mathrm{I} / \mathrm{O}$ effect of the system.

In conclusion, we have developed PaperIO, a paperbased 3D I/O interface using the technology of selective inductive power transmission, and proved its performance through a series of hardware experiments. PaperIO is able to sense the 3D information of power receivers when they are closed to the power transmitter, and convert the input to further control the output of the power transmitter. With the embedded technology, PaperIO is able to provide basic information to support the infrastructure of IoT, such as presence, position, and orientation. The proposed examples show that PaperIO provides a platform for end-users to create their own paper-based sensors and actuators, and connect to the networks to form a new internet of embedded papercraft.

\section{Acknowledgments}

This research was supported by the National Natural Science Foundation of China (No. 60902053, and 61272497).

\section{References}

[1] "Itu internet reports 2005: The internet of things."

[2] D. De Roeck, K. Slegers, J. Criel, M. Godon, L. Claeys, K. Kilpi, and A. Jacobs, "I would diyse for it!: A manifesto for do-ityourself internet-of-things creation," Proc. 7th Nordic Conference on Human-Computer Interaction: Making Sense Through Design, NordiCHI '12, pp.170-179, New York, NY, USA, 2012.

[3] D. Holman and R. Vertegaal, "Organic user interfaces: Designing computers in any way, shape, or form," Commun. ACM, vol.51, no.6, pp.48-55, June 2008.

[4] F. Kaplan and P. Jermann, "Papercomp 2010: First international workshop on paper computing," Proc. 12th ACM International Conference Adjunct Papers on Ubiquitous Computing, Ubicomp '10, pp.507-510, 2010

[5] K. Zhu, H. Nii, O.N.N. Fernando, J.T.K.V. Koh, K. Aue, and A.D. Cheok, "Designing interactive paper-craft systems with selective inductive power transmission," Interacting with Computers, vol.25, no.2, pp.143-153, 2013.

[6] M. Back, J. Cohen, R. Gold, S. Harrison, and S. Minneman, "Listen reader: An electronically augmented paper-based book," Proc. SIGCHI Conference on Human Factors in Computing Systems, CHI '01, pp.23-29, New York, NY, USA, 2001.

[7] Y. Huang, M.D. Gross, E.Y.L. Do, and M. Eisenberg, "Easigami: A reconfigurable folded-sheet tui," TEI '09: Proc. 3rd International Conference on Tangible and Embedded Interaction, pp.107-112, New York, NY, USA, 2009.

[8] M. Coelho, L. Hall, J. Berzowska, and P. Maes, "Pulp-based computing: A framework for building computers out of paper," Proc. 27th International Conference Extended Abstracts on Human Factors in Computing Systems, CHI '09, pp.3527-3528, 2009.

[9] B. Lahey, A. Girouard, W. Burleson, and R. Vertegaal, "Paperphone: Understanding the use of bend gestures in mobile devices with flexible electronic paper displays," Proc. 2011 Annual Conference on Human Factors in Computing Systems, CHI '11, pp.1303-1312, New York, NY, USA, 2011.

[10] G. Saul, C. Xu, and M.D. Gross, "Interactive paper devices: Enduser design and fabrication," Proc. Fourth International Conference on Tangible, Embedded, and Embodied Interaction, TEI '10, pp.205-212, New York, NY, USA, 2010.

[11] L. Buechley, M. Eisenberg, J. Catchen, and A. Crockett, "The lilypad arduino: Using computational textiles to investigate engagement, aesthetics, and diversity in computer science education," Proc. SIGCHI Conference on Human Factors in Computing Systems, CHI '08, pp.423-432, New York, NY, USA, 2008.

[12] J. Qi and L. Buechley, "Electronic popables: Exploring paper-based computing through an interactive pop-up book," TEI '10: Proc. Fourth International Conference on Tangible, Embedded, and Embodied Interaction, pp.121-128, New York, NY, USA, 2010.

[13] N. Koizumi, K. Yasu, A. Liu, M. Sugimoto, and M. Inami, "Animated paper: A moving prototyping platform," Adjunct Proceedings 23rd Annual ACM Symposium on User Interface Software and Technology, UIST '10, pp.389-390, New York, NY, USA, 2010.

[14] K. Zhu and S. Zhao, "Autogami: A low-cost rapid prototyping toolkit for automated movable paper craft," Proc. SIGCHI Conference on Human Factors in Computing Systems, CHI '13, pp.661670, New York, NY, USA, 2013.

[15] "Ltspice iv." http://www.linear.com/ 


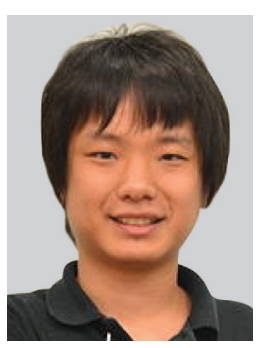

Kening Zhu is an assistant professor in School of Creative Media, City Unversity of Hong Kong. Kening's research interests cover various topics on human-computer interaction (HCI), including interaction design, paper computing, and rapid prototyping. His research focuses on theoretical analysis and technical toolkit for technology-enhanced movable paper craft.

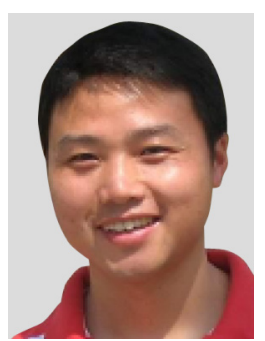

Rongbo Zhu is currently an Associate Professor in College of Computer Science of SouthCentral University for Nationalities, China. He received the $\mathrm{Ph} . \mathrm{D}$. degree in communication and information systems from Shanghai Jiao Tong University, China, in 2006. Dr. Zhu has published over 50 papers in international journals and conferences in the areas of wireless networks and mobile computing. He is the Associate Editor of International Journal of Radio Frequency Identification Technology and Applications. Dr. Zhu is a member of the ACM, IEEE and IEICE.

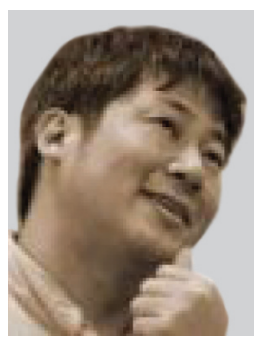

Hideaki Nii got his Ph.D. from University of Electro-communications in 2008. Now he is a researcher at IIJ Innovation Institute, Japan. He was an Assistant Professor of The University of Tokyo from October 2006 to March 2009, and Senior Assistant Professor of Keio University from April 2009 to March 2010.

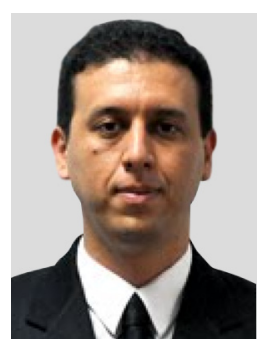

Hooman Samani is Director of the AIART Lab (Artificial Intelligence and Robotics Technology Laboratory) and an Assistant Professor at the Department of Electrical Engineering, College of Electrical Engineering and Computer Science, National Taipei University, Taiwan. He was awarded a $\mathrm{PhD}$ degree from the Graduate School for Integrative Science and Engineering, Electrical \& Computer Engineering Department, National University of Singapore.

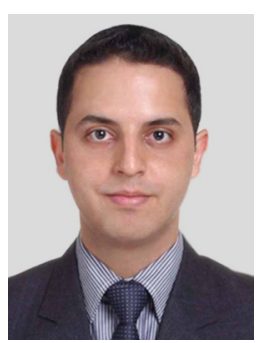

Borhan (Brian) Jalaeian is currently a Ph.D. candidate in the Bradley Department of Electrical and Computer Engineering at Virginia Tech. He is also a Masters student in the Grado Department of Industrial System Engineering concentrating on Operation Research. He has joined and worked in Complex Network and Security Research (CNSR) group at Virginia Tech as a research assistant since 2011. He is a member of Eta Kappa Nu (HKN), an electrical and computer engineering honor society of the IEEE. He is also a member of IEEE Communication Society. 